\title{
Generalized Clifford-Severi Inequality and the Volume of Irregular Varieties
}

\author{
Miguel A. Barja * \\ June 12, 2014
}

\begin{abstract}
We give a sharp lower bound for the self-intersection of a nef line bundle $L$ on an irregular variety $X$ in terms of its continuous global sections and the Albanese dimension of $X$, which we call the Generalized Clifford-Severi inequality. We also extend the result to nef vector bundles and give a slope inequality for fibred irregular varieties. As a byproduct we obtain a lower bound for the volume of irregular varieties; when $X$ is of maximal Albanese dimension the bound is $\operatorname{vol}(X) \geq 2 n ! \chi\left(\omega_{X}\right)$ and it is sharp.
\end{abstract}

\section{Introduction and preliminaries}

The geometry of irregular varieties has been deeply developed in the last 30 years. The seminal results of Green and Lazarsfeld on Generic Vanishing theorems, the generalized Castelnuvo-de Franchis theorem by Catanese and Ran, the systematic use of the Fourier-Mukai techniques associated to the Albanese map and further developments by Lazarsfeld, Ein, Hacon, Chen, Pareschi, Popa and many others have provided a rather complete understanding of birational properties of such varieties.

Another fruitful approach to understanding the geometry of an irregular variety is the study of their continuous linear series. In the case of abelian varieties this approach goes back to Mumford and Kempf (see for example [21], [22]). For the study of irregular surfaces, one of the first instances of a systematic use of them is done in [5]. Later on the work of Pareschi and Popa on continuous global generation and the recent work of Mendes-Lopes, Pardini and Pirola on Brill-Noether theory and continuous families of divisors gives a deep understanding of their geometry in higher dimensions (see [17], [18], and [19]).

In the study of biregular geometry of varieties, inequalities relating the degree and the number of global sections of a line bundle play a special role. This is the goal of our main theorem. In order to stating it, we need to introduce some notation. Consider an irregular variety $X$ and a non trivial map $a: X \longrightarrow A$ to an abelian variety. We say that $X$ is of maximal a-dimension if $\operatorname{dim} a(X)=\operatorname{dim} X$. We associate to any nef line bundle $L$ on $X$ two invariants. One of them is $\delta(L)=\frac{2 r(L)}{2 r(L)-1}$ (see Definition 2.9), a real number between 2 an 1 , where $r(L)$ is the degree of subcanonicity of $L$, the minimal $r$ such that $L \preceq r K_{X}$ (numerically). The second one is $h_{a}^{0}(L)$, the continuous rank of $L$, i.e., the rank of the Fourier-Mukai transform of $L$ with respect to the map $a$, i.e. the minimal value of $h^{0}\left(X, L \otimes a^{*} \alpha\right)$ for $\alpha \in \widehat{A}$. We also will consider $W$ and $M=L-W$, the continuous fixed part and the continuous moving part of $L$, respectively, where $W$ is the common base component of the linear systems $\left|L \otimes a^{*} \alpha\right|$, for $\alpha$ general (see Definition 3.1). With this notation, we can state

*Partially supported by MICINN-MTM2009-14163-C02-02/FEDER, MINECO-MTM2012-38122-C03-01 and by Generalitat de Catalunya 2005SGR00557. 


\section{Main Theorem (Generalized Clifford-Severi Inequality)}

Let $X$ be a smooth, projective variety of dimension $n$, over an algebraically closed field of characteristic 0 . Let $a: X \longrightarrow A$ be a nontrivial map to an Abelian variety and let $L \in \operatorname{Pic}(X)$ be a nef line bundle.

(i) If $X$ is of maximal a-dimension then

$$
L^{n} \geq \delta(L) n ! h_{a}^{0}(L) .
$$

In particular, if $L \preceq K_{X}$, then $L^{n} \geq 2 n ! h_{a}^{0}(L)$.

(ii) Assume $n>\operatorname{dim} a(X)=k \geq 1$ and let $M$ be the continuous moving part of $L$. If $M$ is a-big then

$$
L^{n} \geq \delta(L) k ! h_{a}^{0}(L)
$$

(iii) Assume that $n>\operatorname{dim} a(X)=k \geq 1$ and that $L$ is a-big. Then

$$
L^{n} \geq k ! h_{a}^{0}(L)
$$

We can see this theorem as a wide generalization of the classical Severi inequality for surfaces of maximal Albanese dimension:

$$
K_{S}^{2} \geq 4 \chi\left(\omega_{S}\right)
$$

This inequality was stated by Severi in the 30's ([28]). Many years later, Catanese ([4]) showed a gap in the proof and proposed the inequality as a conjecture. Manetti gave a proof of the conjecture in the case $K_{S}$ ample, together with a profound analysis of the positivity properties of $\Omega_{S}^{1}([15])$. His approach provides further developments and refinements (see [16] and [30]).

The key argument for a complete proof of the Severi inequality without extra hypotheses is given by Pardini ([24], [17]). She deduces the inequality from another well known one: the slope inequality for fibred surfaces ([29], [9]), by using in a quick and clever way the property of being of maximal Albanese dimension. This method, which we call Pardini's covering trick, is completely general, and allows to apply a general principle: given an inequality verified by any maximal Albanese dimension variety you can remove all the numerical data involving lower dimensional subvarieties and obtain a new inequality.

In the present paper we generalize the Severi inequality by an induction argument on the dimension of $X$. For this, we combine three different ingredients:

- A suitable version of Xiao's method for fibrations reducing the problem to étale covers and lower dimensional varieties (induction step). This is done in subsection 5.1.

- The analysis of the behavior of continuous linear series on $X$, i.e., $|L \otimes \alpha|$ for $\alpha \in \widehat{A}$. It allows to assume good behavior of the linear system on an étale covering of $X$. This is done in Section 3.

- The use of Pardini's covering trick $([24])$ to remove unnecessary invariants. This is done in subsection 5.2 .

The initial step of the induction process (the case of curves) is just a continuous version of Clifford's Lemma which we can also consider as a 1-dimensional version of Severi inequality. That's the reason why we add Clifford in the name of the inequality.

There are several particular cases of the main result of independent interest, which are introduced in Chapter 4. Here we present the more relevant ones. The first one is an extension to vector bundles

Corollary A (Generalized Clifford-Severi inequality for nef Vector Bundles) Let $X$ be a projective, smooth variety of dimension $n$, over an algebraically closed field of characteristic 0 . Let $a: X \longrightarrow A$ be $a$ 
nontrivial map to an Abelian variety such that $\operatorname{dim} a(X)=k$. Let $\mathcal{F}$ be a nef vector bundle on $X$ with top Segre class $s(\mathcal{F})$. Assume that $k=n$ or that $\mathcal{F}$ is a-big. Then

$$
s(\mathcal{F}) \geq k ! h_{a}^{0}(\mathcal{F})
$$

When $L=K_{X}$ we obtain the sharp generalization of the classical Severi inequality or, equivalently, a lower bound for the volume of irregular varieties.

Corollary B (Generalized Severi inequality for $K_{X}$ /the volume of irregular varieties) Let $X$ be an irregular, minimal, normal, projective, $\mathbb{Q}$-factorial variety of dimension $n$ over an algebraically closed field of characteristic 0 . Let $X^{\prime}$ be any desingularization of $X$. Then

(i) If $X$ is of maximal Albanese dimension then

$$
K_{X}^{n} \geq 2 n ! \chi\left(\omega_{X^{\prime}}\right)
$$

and this bound is sharp (double covers of abelian varieties).

(ii) If $\operatorname{dim} a l b_{X}(X)=k<n$ and the continuous moving part $M$ of $K_{X^{\prime}}$ is alb ald $_{X^{\prime}}$-big, then

$$
K_{X}^{n} \geq 2 k ! h_{a l b_{X^{\prime}}}^{0}\left(\omega_{X^{\prime}}\right)
$$

(iii) If $\operatorname{dim} a l b_{X}(X)=k<n$ and $X$ is of general type, then

$$
K_{X}^{n} \geq k ! h_{a l b_{X^{\prime}}}^{0}\left(\omega_{X^{\prime}}\right)
$$

This is not a merely application of Main Theorem but we must translate the problem of working with the Q-Cartier Weil divisor $K_{X}$ on the singular variety $X$ to a suitable line bundle $L$ on a desingularization $X^{\prime}$.

We can understand the previous result as a lower bound for the canonical volume of any smooth irregular variety $X^{\prime}$ of general type, just applying Corollary B to a minimal model. It is not easy to find explicit lower bounds for the canonical volume of general type varieties (see for example [6]) and in general it can be very small. It was conjectured by Reid that for varieties with global differential forms the volume should be high. As far as we know, even for the case of irregular 3-folds few results are known (see for example [7], section 3). The bound given by Corollary B gives a sharp lower bound in the maximal Albanese dimension case, attained by double covers of abelian varieties:

$$
\operatorname{vol}\left(X^{\prime}\right) \geq 2 n ! \chi\left(\omega_{X^{\prime}}\right)
$$

During the final preparation of this work, the author has been informed that the content of Corollary B (i), in the case of minimal Gorenstein varieties, was independently proven by Zhang ([31]). There the strategy of proof relies on applying Pardini's trick to the higher dimensional version of the so called Relative Noether inequality, bounding the linear sections of a nef line bundle on an fibred variety.

Our last application shows as the Clifford-Severi inequality implies a slope inequality for fibred varieties. Given a fibred variety over a smooth curve, $f: X \longrightarrow B$ with general fibre $F$, and a line bundle $L$ on $X$, slope inequalities relate the invariants of $(X, L),\left(F, L_{\mid F}\right)$ and $B$. The best slope inequalities hold when some stability properties hold for $L$ (see [2] for a survey on this topic), and then a slope inequality, which we call $f$-positivity of $L$, follows 


$$
L^{n} \geq n \frac{L_{\mid F}^{n-1}}{h^{0}\left(F, L_{\mid F}\right)} \operatorname{deg} f_{*} L .
$$

The case $X$ of general type and $L=\omega_{f}$ encodes important numerical and geographical properties of the fibration, but $f$-positivity of $\omega_{f}$ is only known for fibred surfaces. In fact, $f$-positivity of $\omega_{f}$ for dimensions less or equal to $n$ implies a weaker inequality for the slope

$$
K_{f}^{n} \geq 2 n ! \chi_{f}
$$

from which one can deduce the Severi inequality for $L=\omega_{X}$ (see [2] Proposition 5.8, for a proof of this statement). We prove a converse, namely, that the Severi inequality for $L=\omega_{f}$ implies the above slope inequality when $b=g(B)=0$ and a slightly weaker result when $b \geq 1$.

Corollary C (Slope inequality) Let $f: X \longrightarrow B$ be a relatively minimal fibration onto a smooth curve $B$ of genus $b$ with general fibre $F$ and $\operatorname{dim} X=n$. Assume that $X$ is of maximal Albanese dimension. Then

(i) If $b=0$ then $K_{f}^{n} \geq 2 n ! \chi_{f}$.

(ii) If $b \geq 1$, then $K_{f}^{n} \geq 2 n !\left[\chi\left(\omega_{f}\right)+h_{a}^{1}\left(\omega_{f}\right)\right] \geq 2 n !\left(\chi\left(\omega_{X}\right)-2 \chi\left(\omega_{B}\right) \chi\left(\omega_{F}\right)\right)$.

The paper is divided as follows. The general set-up is $X$ a smooth, projective variety over an algebraically closed field of characteristic 0 , with a nontrivial map $a: X \longrightarrow A$ to an abelian variety.

In section 2 we study the properties of the invariants $h_{a}^{0}(L)$ and $\delta(L)$ associated to any line bundle $L$ on $X$. Section 3 is devoted to studying the behavior of linear systems under étale Galois covers. Here, the concepts of continuous fixed part and continuous moving part of $L$ play an special role. This analysis is a cornerstone in the proof of the main theorem and provides results of independent interest on linear series on irregular varieties. It turns out that (see Theorems 3.3 and 3.6 for more complete results):

Theorem $\mathbf{D}$ Let $X$ be a smooth, projective variety and let $a: X \longrightarrow A$ be a nontrivial map to an Abelian variety such that $a^{*}: \widehat{A} \longrightarrow \operatorname{Pic}^{0} X$ is injective. Up to composing a blow-up with an étale Galois covering, $\lambda: \widetilde{X} \longrightarrow X$

(i) For any $\alpha \in \widehat{A}$ we have a decomposition: $\lambda^{*}(L \otimes \alpha)=\widetilde{W}+\widetilde{N}_{\alpha}$ where the divisor $\widetilde{W}$ is the fixed component (and does not depend on $\alpha$ ) and the moving part $\widetilde{N}_{\alpha}$ is base point free.

(ii) The map $a \circ \lambda$ factors through the algebraic fibre space induced by the linear system $\left|\lambda^{*} L\right|$. In particular $\operatorname{dim} \phi_{\lambda^{*} L}(\widetilde{X}) \geq \operatorname{dim} a(X)$ and so $\left|\lambda^{*} L\right|$ is generically finite provided $X$ is of maximal a-dimension.

Section 4 is devoted to proving Corollaries A, B and C, and giving some remarks, examples and other applications. In Section 5 we prove the Main Theorem. There, an account of Xiao's method especially adapted to étale Galois covers of fibrations onto $\mathbb{P}^{1}$ is given.

Notations and conventions Varieties are assumed to be smooth, projective, defined over an algebraically closed field $k$ of characteristic 0 , except otherwise stated. We use the notation $L$ for a (Cartier) divisor or its associated line bundle interchangeably, except for the canonical sheaf and divisor which will be denoted by $\omega_{X}$ and $K_{X}$ respectively. We use additive or multiplicative notation interchangeably.

Given an abelian variety $A$ we denote by $\widehat{A}=\operatorname{Pic}^{0}(A)$ its dual abelian variety, by $\widehat{A}_{d}$ the subgroup of its $d$-torsion elements and by $\widehat{A}_{\text {tors }}=\bigcup_{d \in \mathbb{N}} \widehat{A}_{d}$ the set of all its torsion elements.

Given an irregular variety $X$ we set $\operatorname{Pic}^{\tau}(X)$ for the set of numerically torsion line bundles on $X$, i.e., the set of $M$ such that $M^{\otimes r} \in \operatorname{Pic}^{0}(X)$ for some $r \in \mathbb{N}$. 
Given a map $f: X \longrightarrow Y$ (not necessarily surjective) we say that it is generically finite if $X \longrightarrow f(X)$ is. We say that $f$ factors through an algebraic fiber space of dimension $k$ with general fibre $G \operatorname{if} \operatorname{dim} f(X)=k$ and so its Stein factorization decomposes $f=g \circ h: X \longrightarrow Z \longrightarrow Y$ where $Z$ is normal of dimension $k, g$ is an algebraic fiber space and $G$ is a general fibre of $g$. As usual, an algebraic fiber space of dimension 1 will be called a fibration.

We will use $\equiv$ for numerical equivalence and given two divisors $D_{1}, D_{2}$ we denote $D_{1} \preceq D_{2}$ if $D_{2}-D_{1}$ is pseudoeffective, i.e., it is a limit of (real) effective divisors or, equivalently, its product with arbitrary nef line bundles is nonnegative.

Acknowledgements The author wants to thank Rita Pardini, Lidia Stoppino, Gian Pietro Pirola, Joan Carles Naranjo, Margarida Mendes-Lopes and Martí Lahoz for fruitful conversations and encouragement during the preparation of this work. The author also wants to thank the anonymous referee for several suggestions to improve the presentation and proofs and for pointing out several inaccuracies in the first version of the paper.

\section{The continuous rank and the subcanonicity index}

In this section we introduce the continuous rank of any coherent sheaf on irregular varieties, and the subcanonicity degree of a line bundle on a variety. The fundamental properties of these two numbers are that both behave well under algebraic equivalence, blow-ups, étale Galois coverings and hyperplane sections.

Given a line bundle on an irregular variety $X$, the behavior of the continuous system given by $|L \otimes \alpha|$ is studied in [19] and in [18]. Our interest relies on general elements in the continuous family.

Definition 2.1. Let $X$ be an irregular variety and $\mathcal{F}$ a coherent sheaf on $X$. Let $A$ be an abelian variety and $a: X \longrightarrow A$ a nontrivial map. Define

$$
h_{a}^{i}(\mathcal{F}):=\min \left\{h^{i}\left(X, \mathcal{F} \otimes a^{*} \alpha\right) \mid \alpha \in \operatorname{Pic}^{0}(A)\right\}
$$

$h_{a}^{0}(\mathcal{F})$ will be called the continuous rank of $\mathcal{F}$.

By abuse of notation, if the map is clear by the context, we will usually write $\alpha$ instead of $a^{*} \alpha$.

Remark 2.2. If $R S_{a}$ is the integral Fourier-Mukai functor associated to the map a (see [25]), we have that $h_{a}^{i}(\mathcal{F})=\operatorname{rank} R S_{a}^{i}(\mathcal{F})$.

Definition 2.3. If $a: X \longrightarrow A$ is a map from $X$ to an abelian variety, we will say that $X$ is of maximal a-dimension if $\operatorname{dim} X=\operatorname{dim} a(X)$. When $A=\operatorname{Alb}(X)$ and $a=\operatorname{alb}_{X}$ the definition corresponds to the classical notion of a maximal Albanese dimension variety.

Example 2.4. As simple examples related to line bundles $L \in \operatorname{Pic}(X)$ on smooth $X$, we have

- If $L=K_{X}+D$ with $D$ nef, and $X$ is of maximal $a$-dimension, then $h_{a}^{0}(L)=\chi(X, L)$ by the Generic Vanishing theorem given by Pareschi-Popa (Theorem B in [27]). If $\operatorname{dim} \operatorname{alb}(X)=\operatorname{dim} X-1$ then we still have $h_{a}^{0}(\mathcal{L}) \geq h_{a}^{0}(\mathcal{L})-h_{a}^{1}(\mathcal{L})=\chi(X, \mathcal{L})$.

- The same holds for any $G V$-sheaf, like higher direct images of relative dualizing sheaves (see also [27]).

- If $L=K_{X}+D$ with $D$ big and nef, then $h_{a}^{0}(L)=\chi(X, L)=h^{0}(X, L)$ by Kawamata-Viehweg vanishing theorem.

Remark 2.5. The author was informed by Pirola that using the arguments of [19] and [18] and bounding the obstructions to deforming a global section of $L$, the following inequality holds: $h_{a}^{0}(L) \geq h^{0}(X, L)-h^{1}(X, L)$ (unpublished). 
Remark 2.6. The Severi inequality can be restated as $K_{S}^{2} \geq 4 h_{a l b_{S}}^{0}\left(\omega_{S}\right)$.

Let's see now a non trivial example

Proposition 2.7. Let $f: X \longrightarrow B$ be a fibration onto a smooth, projective curve of genus $b$, with general fibre $F$. Assume that $X$ is smooth of general type and of maximal Albanese dimension. Let $a=a l b_{X}$. Then

$$
h_{a}^{0}\left(\omega_{f}\right)=h_{a}^{1}\left(\omega_{f}\right)+\chi\left(X, \omega_{f}\right)=h_{a}^{1}\left(\omega_{f}\right)+\chi\left(X, \omega_{X}\right)-2 \chi\left(B, \omega_{B}\right) \chi\left(F, \omega_{F}\right) .
$$

If $b=0,1$ we have in fact that $h_{a}^{0}\left(\omega_{f}\right)=\chi\left(X, \omega_{X}\right)-(2 b-2) \chi\left(F, \omega_{F}\right) \geq \chi\left(X, \omega_{X}\right)$.

Proof. Let $A=A l b(X)$. The subset of $\operatorname{Pic}^{0}(A)$ where $h^{0}\left(X, \omega_{f} \otimes a^{*} \alpha\right)$ takes its minimum value is an open set, so it is enough to compute this value for a general $\alpha \in \widehat{A}_{\text {tors. }}$. In this case $R^{i} f_{*}\left(\omega_{f} \otimes \alpha\right)$ is locally free on $B$ ([12]), of rank $h^{i}\left(F, \omega_{F} \otimes \alpha\right)$, which is zero for $i \geq 1$ by generic vanishing applied to the map $\bar{a}: F \hookrightarrow X \longrightarrow A$ (clearly $F$ is of maximal $\bar{a}$-dimension). Hence $H^{i}\left(X, \omega_{f} \otimes \alpha\right)=H^{i}\left(B, f_{*}\left(\omega_{f} \otimes \alpha\right)\right)=0$ for $i \geq 2$, and the result follows. Observe that the equality $\chi\left(X, \omega_{f}\right)=\chi\left(X, \omega_{X}\right)-2 \chi\left(B, \omega_{B}\right) \chi\left(F, \omega_{F}\right)$ holds since for any line bundle on $X$ the equality $\chi(X, L+k F)=\chi(X, L)+k \chi\left(F, L_{\mid F}\right)$ follows by induction on $k$.

When $b=0,1$, consider the étale covering given by $\alpha, g: \bar{X} \longrightarrow X$. We can choose $\alpha$ of prime order $p$ such that $\bar{a}^{*} \alpha \neq \mathcal{O}_{F}$, and so $\bar{a}^{*} \alpha^{\otimes i} \neq \mathcal{O}_{F}$ for all $1 \leq i \leq p-1$. Hence, the induced map $\bar{f}=f \circ g: \bar{X} \longrightarrow B$ is a fibration (the fibres are the connected étale cover of $F$ given by $\left.\bar{a}^{*} \alpha \neq \mathcal{O}_{F}\right)$. Then $f_{*}\left(\omega_{f} \otimes \alpha\right)$ is a direct summand of $\bar{f}_{*}\left(\omega_{\bar{f}}\right)$, and so it is a nef vector bundle on $B$, since $\bar{f}_{*}\left(\omega_{\bar{f}}\right)$ is ([11]). If $b=0$ this proves $H^{1}\left(X, \omega_{f} \otimes \alpha\right)=H^{1}\left(B, f_{*}\left(\omega_{f} \otimes \alpha\right)\right)=0$. If $b=1$, we also obtain that $H^{1}\left(X, \omega_{f} \otimes \alpha\right)=H^{1}\left(B, \omega_{X} \otimes \alpha\right)=0$ by generic vanishing.

Finally let us see how $h_{a}^{i}$ behaves under étale covers, blow-ups and hyperplane sections.

Proposition 2.8. Let $X$ be a smooth, projective irregular variety and let $a: X \longrightarrow A$ be a nontrivial map to an abelian variety $A$ such that $a^{*}: \widehat{A} \longrightarrow \operatorname{Pic}^{0} X$ is injective. Take $L \in \operatorname{Pic}(X)$.

(i) Let $\mu: \widetilde{A} \longrightarrow A$ a degree $m$ isogeny and consider the base change diagram

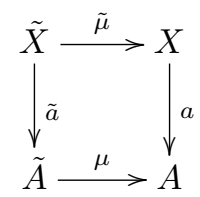

Then $h_{\tilde{a}}^{i}\left(\tilde{\mu}^{*}(L)=m h_{a}^{i}(L)\right.$.

(ii) If $\sigma: X^{\prime} \longrightarrow X$ is a blow-up, then $h_{a}^{0}\left(\sigma^{*} L\right)=h_{a}^{0}(L)$.

(iii) If $H$ is a smooth divisor on $X$ not contracted by a, such that $H-L$ is nef, then $h_{a}^{0}\left(L_{\mid H}\right) \geq h_{a}^{0}(L)$.

Proof. (i) Let $N=\operatorname{Ker} \mu \subseteq \hat{A}$, which is of order $m$. We have that $\widetilde{X}$ is connected since $a^{*}$ is injective and $\tilde{\mu}_{*}\left(\mathcal{O}_{\tilde{X}}\right)=\bigoplus_{\gamma \in N} a^{*}(\gamma)$. Let $Z_{i} \subsetneq \hat{A}$ be the jumping locus of the value $h^{i}\left(X, a^{*}(\alpha)\right)$, which is a proper closed set. For any $\beta=\mu^{*}(\alpha) \notin \mu^{*}\left(Z_{i}\right)$ we have

$$
h^{i}\left(\tilde{X}, \tilde{\mu}^{*} L \otimes \tilde{a}^{*} \beta\right)=\bigoplus_{\gamma \in N} h^{i}\left(X, L \otimes a^{*}(\alpha \otimes \gamma)\right)=m h_{a}^{i}(L)
$$

since $\alpha \otimes \gamma \notin Z_{i}$ for all $\gamma \in N$.

(ii) Obvious.

(iii) For $\alpha \in \widehat{A}$ we can consider the exact sequence 


$$
0 \longrightarrow \mathcal{O}_{X}((L-H) \otimes \alpha) \longrightarrow \mathcal{O}_{X}(L \otimes \alpha) \longrightarrow \mathcal{O}_{H}\left(L_{H} \otimes \alpha\right) \longrightarrow 0
$$

Taking cohomology we have $h^{0}(X,(L-H) \otimes \alpha)=h^{n}\left(X, K_{X}+(H-L) \otimes \alpha^{-1}\right)=0$ for $\alpha$ general, by the generic vanishing theorem (cf. Theorem $\mathrm{B}$ in [27]), since we are asuming $H-L$ is nef.

Let us consider now the second invariant we need. As in the case of curves, the smaller is the degree of a nef line bundle, the bigger is the ratio between its degree and its global sections. The best behavior, given by Clifford's lemma, holds for subcanonical line bundles. We introduce now an invariant which measures exactly this relation in terms of $r$-subcanonicity. More concretely we have

Definition 2.9. Given a nef line bundle $L$ we define

(i) $r(L)=\inf \left\{r \geq 1 \mid L \preceq r K_{X}\right\}$ (degree of subcanonicity of $L$ ). Note that if this set is empty, then $r(L)=\infty$. We will say that $L$ is subcanonical if $r(L)=1$.

(ii) $\delta(L)=\frac{2 r(L)}{2 r(L)-1}$.

Remark 2.10. The following are easy properties of $\delta$ :

1. $\delta(L)$ is a decreasing function of $r$, varying between 2 and $1 . \delta(L)=2$ if and only if $L$ is (numerically) subcanonical and $\delta(L)=1$ if and only if $r(L)=\infty$.

2. $\delta(L)$ is a decreasing function of $L$, i.e., if $L_{1} \preceq L_{2}$ then $\delta\left(L_{1}\right) \geq \delta\left(L_{2}\right)$.

3. $\delta(L)$ increases by hyperplane section, i.e., if $M$ is a smooth section of a nef line bundle then $\delta\left(L_{\mid M}\right) \geq$ $\delta(L)$.

4. If $\mu: \widetilde{X} \longrightarrow X$ is an étale Galois covering and $\widetilde{L}=\mu^{*} L$, then $\delta(\widetilde{L})=\delta(L)$.

5. $\delta(L)$ increases by blow-up, i.e., if $\sigma: X^{\prime} \longrightarrow X$ is any blow-up, then $\delta\left(\sigma^{*}(L)\right) \geq \delta(L)$.

6. If $X$ is a variety of general type, then $K_{X}$ is big and hence for all $L$ there exists an $r$ such that $r K_{X}-L$ is effective. Then we always have $\delta(L)>1$. The case $X=A$ an abelian variety is just the opposite: $\delta\left(\mathcal{O}_{A}\right)=2$ and for all nef $L \neq \mathcal{O}_{A}$ we have $\delta(L)=1$.

\section{Some properties of continuous linear systems}

Let $a: X \longrightarrow A$ be a nontrivial map to an abelian variety and let $L \in \operatorname{Pic}(\mathrm{X})$. We are going to study the geometry of the continuous linear systems $|L \otimes \alpha|$ for $\alpha$ general. A good presentation and an analysis of the generic base loci of the main continuous system associated to $L$ is developed in [19] and [18]. Here we consider two related problems: the continuous resolution of base points of a continuous linear system (Theorem 3.2), and the behavior of a general $|L \otimes \alpha|$ up to an étale covering (Theorem 3.5).

It is well known that there exists a nonempty open set

$$
U \subseteq U_{a, L}=\left\{\alpha \in \widehat{A} \mid h^{0}(X, L \otimes \alpha)=h_{a}^{0}(L)\right\}
$$

such that for $\alpha \in U$, if we consider the decomposition $L \otimes \alpha=W_{\alpha}+N_{\alpha}$ into its fixed and moving part respectively, then the divisors $W_{\alpha}$ belong to the same algebraic class, and the same occurs with the divisors $N_{\alpha}$. This is basically the construction given in [1], section 5.1, for the canonical line bundle and $\operatorname{Pic}^{0}(X)$, but it holds in general. Roughly speaking, consider in $X \times \widehat{A}$ the closed set $\mathcal{D}=\{(p, \alpha) \mid p \in \operatorname{Bs}(|L \otimes \alpha|)\}$. When 
$|L \otimes \alpha|$ has a divisorial base component, $\mathcal{D}$ has a codimension 1 component which is dominant with respect to the second projection. On an open set $U$ of $\operatorname{Pic}^{0}(\mathrm{X})$ its fibres are algebraically equivalent.

We define now the continuous moving part and the continuous fixed part of $L$ as follows. Consider first the evaluation map

$$
e v_{U}:=\oplus e v_{\alpha}: \bigoplus_{\alpha \in U} H^{0}(X, L \otimes \alpha) \otimes \alpha^{-1} \longrightarrow L .
$$

We have that $\operatorname{Imev}_{U}=\mathcal{I}_{U} \otimes L$, where $\mathcal{I}_{U}$ is an ideal sheaf. In the proof of Lemma 3.2 we will see that this sheaf does not depend on the chosen open set $U$ verifying the conditions above. Consider its decomposition

$$
\mathcal{I}_{U}=\mathcal{O}_{X}(-W) \otimes \mathcal{I}_{B}
$$

with $\operatorname{codim}_{X} B \geq 2$.

Definition 3.1. $\quad$ (i) $W$ is the continuous fixed part of $L$.

(ii) $M=L-W$ is the continuous moving part of $L$.

Set $\operatorname{Imev}_{\alpha}=\mathcal{I}_{\alpha} \otimes L \otimes \alpha$ where $\mathcal{I}_{\alpha}=\mathcal{O}\left(-W_{\alpha}\right) \otimes \mathcal{I}_{B_{\alpha}}$ with $\operatorname{codim}_{X}\left(B_{\alpha}\right) \geq 2$. Observe that, by construction we have

$$
\mathcal{I}_{\alpha} \otimes L \otimes \alpha \subseteq M \otimes \alpha \subseteq L \otimes \alpha .
$$

Hence we have that

$$
h_{a}^{0}(L)=h_{a}^{0}(M) .
$$

Following Pareschi and Popa ([26]), recall that a line bundle $L$ on $X$ is continuously globally generated with respect to the map $a$ if the continuous evaluations maps $e v_{V}$ defined above are surjective for all non-empty open sets $V \subseteq \widehat{A}$. First of all we have

Lemma 3.2. With the previous notation, the sheaf $\mathcal{F}=\operatorname{Im}\left(e v_{U}\right)$ is continuously globally generated with respect to $a$.

Proof. This is the content of Remark 4.2 in [1]. To sketch a proof observe that given a point $p \in X$, if there exists a nonempty open subset $V \subseteq U$ such that $p$ is a base point of the linear systems $|L \otimes \alpha|$ for all $\alpha \in V$, then $p$ is also a base point of these linear systems for all $\alpha \in U$. Indeed, all the sections in $H^{0}(X, L \otimes \alpha)$, $\alpha \in U$, are limits of those with $\alpha \in V$, since on $U$ the dimensions $h^{0}(X, L \otimes \alpha)$ are constant.

Hence, for any open set $V \subseteq \widehat{A}$ we have

$$
\operatorname{Im}\left(e v_{V}\right) \supseteq \operatorname{Im}\left(e v_{V \cap U}\right)=\operatorname{Im}\left(e v_{U}\right)=\mathcal{F} .
$$

Finally, observe that by construction we have for any $\alpha \in U$

$$
\mathcal{I}_{\alpha} \otimes(L \otimes \alpha) \subseteq \mathcal{F} \otimes \alpha \subseteq L \otimes \alpha
$$

and hence $H^{0}(X, \mathcal{F} \otimes \alpha)=H^{0}(X, L \otimes \alpha)$ and so we have $\operatorname{Im}\left(e v_{U, L}\right)=\operatorname{Im}\left(e v_{U, \mathcal{F}}\right)$.

Now we are going to see that the continuous fixed and moving parts of $L$ behave as the linear ones up to a suitable étale covering. 
Theorem 3.3. Let $X$ be a smooth projective irregular variety and let $a: X \longrightarrow A$ be a nontrivial map to an abelian variety, such that $a^{*}: \widehat{A} \longrightarrow \mathrm{Pic}^{0} X$ is injective. Then, up to a blow-up $\sigma$ and an étale Galois covering (more concretely, a base change by a multiplication map) $\mu$

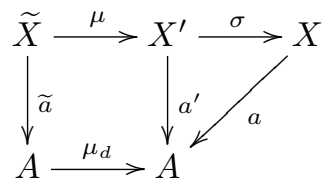

we have that for all $\alpha \in \widehat{A}$

$$
\lambda^{*}(L \otimes \alpha)=\widetilde{W}+\widetilde{N}_{\alpha}
$$

is the decomposition in the fixed and moving divisor, $\lambda=\mu \circ \sigma$, the linear system $\left|\tilde{N}_{\alpha}\right|$ is base point free and the divisor $\widetilde{W}$ does not depend on $\alpha$.

Proof. Consider a blow-up $\sigma: X^{\prime} \longrightarrow X$ such that $\sigma^{*}\left(\mathcal{O}_{X}(-W) \otimes \mathcal{I}_{B}\right)=\mathcal{O}_{X^{\prime}}\left(-W^{\prime}\right)$. If we set $L^{\prime}=\sigma^{*} L$, then we have that $\sigma^{*} \mathcal{F}=L^{\prime}\left(-W^{\prime}\right)$ is continuously globally generated with respect to $a^{\prime}=a \circ \sigma$. Then we can apply a result of Debarre (cf. [10] Proposition 3.1): there exists an étale Galois covering $\mu: \widetilde{X} \longrightarrow X^{\prime}$ which we can assume is induced by a multiplication map on $A$, such that for any $\alpha \in \widehat{A}$, the line bundles $\widetilde{L}\left(-\mu^{*} W^{\prime}\right) \otimes \alpha=\mu^{*} L^{\prime}\left(-W^{\prime}\right) \otimes \alpha$ are globally generated. Hence, if we set $\widetilde{W}=\mu^{*} W^{\prime}$ we have the statement. The proof of Debarre is for alb $\operatorname{los}_{X}$ but it also works for any map a such that $a^{*}$ is injective (hence $\tilde{X}$ is connected).

Remark 3.5. Observe that in the previous construction we have the following properties

- For all $\alpha \in \widehat{A}$ the line bundles $\widetilde{N}_{\alpha}$ on $\widetilde{X}$ are algebraically equivalent.

- $h_{\widetilde{a}}^{0}\left(\widetilde{N}_{\alpha}\right)=h_{\widetilde{a}}^{0}(\widetilde{L})=(\operatorname{deg} \mu) h_{a^{\prime}}^{0}\left(L^{\prime}\right)=(\operatorname{deg} \mu) h_{a}^{0}(M)=(\operatorname{deg} \mu) h_{a}^{0}(L)$.

- For all $l \geq 1$ we have $h^{0}\left(\tilde{X}, \tilde{N}_{\alpha}^{\otimes l}\right)=(\operatorname{deg} \mu) h^{0}\left(X,(M \otimes \alpha)^{\otimes l}\right)$.

- Since $\tilde{N}_{\alpha}$ are base point free, if $L$ is nef then $(\operatorname{deg} \mu) L^{n}=(\widetilde{L})^{n} \geq\left(\widetilde{N}_{\alpha}\right)^{n}$.

To finish the section we are going to see that given a nef line bundle with continuous sections, the image of the map induced by its linear sections, up to étale base change, factors the map $a$. Hence the dimension of the image of $X$ through the linear system $|L|$ is bounded by the $a$-dimension of $X$. In particular, it is maximal when $X$ is of maximal $a$-dimension. This will be a crucial point in the proof of the Main Theorem.

In order to do this, consider the following notation. Given a line bundle $L$ with nontrivial sections consider the morphism $\psi_{L}: X^{\prime} \longrightarrow \mathbb{P}^{m}$ it induces on a suitable blow-up $X^{\prime}$ of $X$. We denote by

$$
\phi_{L}: X^{\prime} \longrightarrow Z_{L}
$$

the algebraic fibre space induced by $\psi_{L}$.

Theorem 3.6. Let $X$ be a smooth n-dimensional variety and $a: X \longrightarrow A$ a nontrivial map to an abelian variety such that $a^{*}: \widehat{A} \longrightarrow \operatorname{Pic}^{0} X$ is injective. Let $k=\operatorname{dim} a(X)$. Let $L \in \operatorname{Pic} X$, such that $h_{a}^{0}(L) \neq 0$. Let $M$ be its continuous moving part. Consider the previous notation of this section and the map $\lambda$ given by Theorem 3.3. Then

(i) There exists a factorization $\widetilde{a}=\phi_{\widetilde{L}} \circ a^{\prime}$, where $a^{\prime}: Z_{\widetilde{L}} \longrightarrow A$. In particular $\operatorname{dim} \psi_{\widetilde{L}}(\widetilde{X}) \geq k$. Moreover, for all $\alpha \in \widehat{A}$ we have that $N_{\alpha} \in \phi_{\widetilde{L}}^{*} \operatorname{Pic} Z_{\widetilde{L}}$. 
(ii) The linear system $|\widetilde{L}|$ induces a generically finite map provided one of the following conditions hold

- $|L|$ induces a generically finite map.

- $X$ is of maximal a-dimension.

- $M$ is a-big.

Proof. (i) Let $T$ be a general (connected) fibre of $\phi_{\widetilde{L}}$, and let $R \in \operatorname{Pic}\left(Z_{\widetilde{L}}\right)$ such that $\widetilde{N}_{0}=\phi_{\widetilde{L}}^{*}(R)$. By Remark 3.4 , if $h_{a}^{0}(L) \neq 0$ then $h_{\widetilde{a}}^{0}\left(\widetilde{N}_{0}\right) \neq 0$ and hence for all $\alpha \in \widehat{A}$ we have

$$
h^{0}\left(\widetilde{X}, \widetilde{N}_{0} \otimes \widetilde{a}^{*} \alpha\right) \neq 0 .
$$

By projection formula we have that $h^{0}\left(Z_{\widetilde{L}}, R \otimes\left(\phi_{\widetilde{L}}\right)_{*}\left(\widetilde{a}^{*} \alpha\right)\right) \neq 0$ for all $\alpha \in \widehat{A}$. The sheaf $\left(\phi_{\widetilde{L}}\right)_{*}\left(\widetilde{a}^{*} \alpha\right)$ is torsion free of rank $h^{0}\left(T,\left(\widetilde{a}^{*} \alpha\right)_{\mid T}\right)$ and so it must be non zero for all $\alpha$. Since $\left(\widetilde{a}^{*} \alpha\right) \in \operatorname{Pic}^{0}(T)$ this can only happen if $\left(\widetilde{a}^{*} \alpha\right)_{\mid T}=\mathcal{O}_{T}$. Hence the natural composition map

$$
\widehat{A} \longrightarrow \operatorname{Pic}^{0}(\tilde{X}) \longrightarrow \operatorname{Pic}^{0}(T)
$$

is zero. Dualizing we obtain that for general $T$ the map $\widetilde{a}$ contracts $T$ to a point.

The rest of the statement follows immediately from this factorization.

(ii) If $|L|$ induces a generically finite map clearly so does $|\widetilde{L}|$. By (i) the same holds if $X$ is of maximal $a$-dimension $(k=n)$. For the rest, observe that the fibres $G^{\prime}$ of the map $a \circ \lambda$ are just disconnected copies of $G$. On the other hand, by construction $\widetilde{N}_{0 \mid G^{\prime}}$ is big if and only if $M_{\mid G^{\prime}}$ is, hence we can assume that $\widetilde{N}_{0}$ is $\widetilde{a}$-big. But if $r<n$ this is not possible since the fibres $G^{\prime}$ are covered by those of $\phi_{\widetilde{L}}$.

Remark 3.7. Clearly $a$-bigness of $M$ follows from bigness of $M$ itself, but in general it can be difficult to check. Here we have three sufficient conditions.

- If $L$ is $a$-big (for example, if $L$ itself is big) and $\operatorname{kod}\left(G, W_{\mid G}\right) \leq 0$, then $M a$-is big.

- If $L$ is continuously globally generated in codimension 2 (i.e. $W=\emptyset$ ), then bigness of $M$ is equivalent to bigness of $L$, i.e., $L^{n}>0$.

- Continuous global generation of $L$ outside of the ramification locus of the Albanese map of $X$, is implied by $M$-regularity of $L$ (see [26]).

Remark 3.8. In general $a$-bigness of $L$ does not imply $a$-bigness of its continuous moving part $M$. Take for example $X=S \times Y$ where $S$ is a general type surface with $p_{g}=1, q=0$ and $Y$ is a general type and albanese general type variety of dimension $n-2$, with base point free paracanonical system $N$. Then clearly $L=\omega_{X}$ is big, its continuous fixed part is $Z=\pi_{1}^{*}(D)(D$ the only canonical section of $S)$ and $M=\pi_{2}^{*} N$, the Albanese map of $X$ is just the Albanese map of $Y$ and $M_{\mid G}$ is not big.

\section{The generalized Clifford-Severi Inequality}

In the previous sections we have introduced all the ingredients needed to state and prove our results. All of them are particular instances or corollaries of the Main Theorem in the introduction which we reproduce here.

\section{Theorem 4.1. (Generalized Clifford-Severi Inequality)}

Let $X$ be a smooth, projective variety of dimension $n$, over an algebraically closed field of characteristic 0 . Let $a: X \longrightarrow A$ be a nontrivial map to an Abelian variety and let $L \in \operatorname{Pic}(X)$ be a nef line bundle. 
(i) If $X$ is of maximal a-dimension then

$$
L^{n} \geq \delta(L) n ! h_{a}^{0}(L) .
$$

In particular, if $L \preceq K_{X}$, then $L^{n} \geq 2 n ! h_{a}^{0}(L)$.

(ii) Assume $n>\operatorname{dim} a(X)=k \geq 1$ and let $M$ be the continuous moving part of $L$. If $M$ is a-big then

$$
L^{n} \geq \delta(L) k ! h_{a}^{0}(L) .
$$

(iii) Assume that $n>\operatorname{dim} a(X)=k \geq 1$ and that $L$ is a-big. Then

$$
L^{n} \geq k ! h_{a}^{0}(L) .
$$

The proof of this theorem relies on a suitable use of Xiao's method on étale Galois coverings of $X$ and it is postponed to the next Section.

Remark 4.2. Since $\delta$ is a decreasing function, we do not need to know the exact value of $r(L)$ to obtain an inequality. Hence, part (i) of the theorem can be rephrased as (analogously for (ii)): when $X$ is of maximal $a$-dimension

(i1) For any nef $L$ we have $L^{n} \geq n ! h_{a}^{0}(L)$.

(i2) If $L \preceq r K_{X}(r \geq 1)$, then $L^{n} \geq \frac{2 r}{2 r-1} n$ ! $h_{a}^{0}(L)$.

Remark 4.3. (i) The bound given in 4.1 (i) is sharp for the lowest value of $\delta(L)$ : take $X=A$ an abelian variety and $L$ an ample line bundle on $A$. Also, in general it is asymptotically sharp for general type varieties $X$ and sufficiently ample line bundles $L=m H$, as shown by asymptotic Riemann-Roch theorem when $m \rightarrow \infty$.

(ii) For varieties of non maximal Albanese dimension Theorem 4.1 cannot hold without extra hypothesis as those in (ii) and (iii). Indeed, take $L$ to be the pullback of a line bundle on the Albanese image of $X$. Then $L^{n}=0$ and in general $h_{a l b_{X}}^{0}(L) \neq 0$.

Remark 4.4. As shown in Remark 2.4, the continuous rank $h_{a}^{0}(L)$ is just $\chi(X, L)$ or even $h^{0}(X, L)$ under conditions of positivity of $L$ and of maximal $a$-dimension of $X$, which produce (generic) vanishing of higher cohomology.

We proceed now to prove the corollaries A,B and C stated in the Introduction.

Proof. Corollary $A$. Let $Y=\mathbb{P}_{X}(\mathcal{F}), \pi: Y \longrightarrow X$ the natural projection and $L=\mathcal{O}_{Y}(1)$ the tautological line bundle. Consider the induced map $a_{Y}:=a \circ \pi: Y \longrightarrow A$; we have $\operatorname{dim} a_{Y}(Y)=k$. Observe that $s(\mathcal{F})=\mathcal{O}_{Y}(1)^{n+l-1}$ where $l=\operatorname{rank} \mathcal{F}$.

Let $G^{\prime}$ be the fibre of the algebraic fibre space induced by the Albanese map of $Y$. If $k=n$ then $G^{\prime}=\mathbb{P}^{l-1}$, $L_{\mid G^{\prime}}=\mathcal{O}_{\mathbb{P} l-1}(1)$ and hypothesis of theorem 4.1 (ii) holds. If $k<n$ then $G^{\prime}$ is a projective bundle on $G$. If $\mathcal{F}_{\mid G}$ is big, then the hypothesis of (iii) holds. In both cases we use $\delta(L) \geq 1$.

Proof. Corollary B. Since $X$ is normal we can consider the canonical Weil divisor $K_{X}$ and set $\omega_{X}=\mathcal{O}_{X}\left(K_{X}\right)$ for the associated divisorial sheaf. Consider a desingularization $\sigma: X^{\prime} \longrightarrow X$; since $X$ is minimal it has terminal singularities and we have that $\operatorname{Alb} X=\operatorname{Alb} X^{\prime}=: A$ and $\operatorname{alb}_{X^{\prime}}=\operatorname{alb}_{X} \circ \sigma$ ([3], Ch.2.4). Terminal singularities also give $H^{0}\left(X^{\prime}, \omega_{X^{\prime}}\right) \cong H^{0}\left(X, \sigma_{*} \omega_{X^{\prime}}\right)=H^{0}\left(X, \omega_{X}\right)$ and so $h_{\mathrm{alb}_{X}}^{0}\left(\omega_{X}\right)=h_{\mathrm{alb}_{X^{\prime}}}^{0}\left(\omega_{X^{\prime}}\right)$ and it coincides with $\chi\left(\omega_{X^{\prime}}\right)$ when $X$ is of maximal Albanese dimension.

To avoid working with the singularities of $X$, we are going to show now how to reduce the computation to a suitable line bundle $M$ on $X^{\prime}$ such that $K_{X}^{n} \geq M^{n}$ and $h_{a l b_{X^{\prime}}}^{0}(M)=h_{a l b_{X^{\prime}}}^{0}\left(\omega_{X^{\prime}}\right)$. 
Since the inequalities are invariant through étale covers, by Theorem 3.3 we can consider an extra blow-up and a base change through a multiplication map and assume that $K_{X^{\prime}}=W+M$, where $W$ and $M$ are the continuous fixed divisor and the continuous moving divisor of $K_{X^{\prime}}$ respectively and the linear system $|M|$ is base point free. Moreover, as seen after Definition 3.1, we have that $h_{a l b_{X^{\prime}}}^{0}(M)=h_{a l b_{X^{\prime}}}^{0}\left(\omega_{X^{\prime}}\right)$. Since $|M|$ is base point free we have an induced morphism $\lambda^{\prime}: X^{\prime} \longrightarrow \mathbb{P}^{\prime}:=\mathbb{P}\left(H^{0}\left(X^{\prime}, \omega_{X^{\prime}}\right)^{*}\right)$ such that $M=\lambda^{\prime *} T_{\mathbb{P}^{\prime}}$ where $T_{\mathbb{P}^{\prime}}=\mathcal{O}_{\mathbb{P}^{\prime}}(1)$.

Since the variety $X$ is normal and $\mathbb{Q}$-factorial, we can apply a $\mathbb{Q}$-resolution of base loci for Weil divisors. We claim that there exists a desingularization (which we still call $\left.X^{\prime}\right) \sigma: X^{\prime} \longrightarrow X$, an effective Weil divisor $E_{0}$ on $X$ and an effective $\sigma$-exceptional $\mathbb{Q}$-divisor $E$ on $X^{\prime}$ such that

$$
\sigma^{*}\left(K_{X}-E_{0}\right)-E \sim \mathbb{Q}(\lambda \circ \sigma)^{*} T_{\mathbb{P}}
$$

where $\lambda: X \rightarrow \mathbb{P}\left(H^{0}\left(X, \omega_{X}\right)^{*}\right)$ is the natural map and $\sim_{\mathbb{Q}}$ means $\mathbb{Q}$-linear equivalence. The natural identification $\mathbb{P}\left(H^{0}\left(X^{\prime}, \omega_{X^{\prime}}\right)^{*}\right)=\mathbb{P}\left(H^{0}\left(X, \omega_{X}\right)^{*}\right)$ induces $\lambda^{\prime}=\lambda \circ \sigma$ and so $(\lambda \circ \sigma)^{*} T_{\mathbb{P}}=\left(\lambda^{\prime}\right)^{*} T_{\mathbb{P}^{\prime}}=M$.

Indeed, the claim is an absolute version of Lemma 1.1 in [23]. We sketch here the proof and refer there for details. Consider $E_{0}$ a Weil divisor on $X$ such that $e v: H^{0}\left(X, K_{X}\right) \otimes \mathcal{O}_{X} \longrightarrow \mathcal{O}_{X}\left(K_{X}-E_{0}\right)$ is surjective in codimension 1. Take a positive integer $m$ such that $m\left(K_{X}-E_{0}\right)$ is Cartier. Up to a suitable resolution of singularities and base loci, we obtain a desingularization $\sigma: X^{\prime} \longrightarrow X$ and an effective divisor $\bar{E}$ on $X^{\prime}$ such that the natural morphism $\sigma^{*}\left(\operatorname{Sym}^{m} H^{0}\left(X, K_{X}\right) \otimes \mathcal{O}_{X}\right) \longrightarrow \sigma^{*}\left(m\left(K_{X}-E_{0}\right)\right) \otimes \mathcal{O}_{X^{\prime}}(-\bar{E})$ is surjective. Hence we obtain

$$
\sigma^{*}\left(m\left(K_{X}-E_{0}\right)\right)-\bar{E}=\lambda_{m}^{*} T_{\mathbb{P}\left(\left(\operatorname{Sym}^{m} H^{0}\left(K_{X}\right)\right)^{*}\right)}=m(\lambda \circ \sigma)^{*} T_{\mathbb{P}\left(H^{0}\left(X, K_{X}\right)^{*}\right)}=m M
$$

where $\lambda_{m}$ is the natural morphism $X^{\prime} \longrightarrow \mathbb{P}\left(\left(\operatorname{Sym}^{m} H^{0}\left(K_{X}\right)\right)^{*}\right)$. The claim then follows just dividing by $m$ and defining $E:=\frac{1}{m} \bar{E}$.

In order to conclude the proof of the corollary, just observe that $M$ and $K_{X}$ are nef and that $E_{0}$ and $E$ are effective. Hence

$$
K_{X}^{n}=\left(\sigma^{*} K_{X}\right)^{n} \geq M^{n} .
$$
ical.

Then the results follow from Main Theorem applied to $\left(X^{\prime}, M\right)$, using that $\delta(M)=2$ since it is subcanon-

Remark 4.5. Irregular, general type varieties of Albanese dimension $k$, with $\operatorname{vol}(X)<2 k ! \chi\left(\omega_{X}\right)$ seem to have strong restrictions, if they exist. At least when the minimal model $X$ is Gorenstein, the albanese map of $X$ must factor through a fibration with general fibre $G$ of dimension $l \geq 1$ such that $K_{G}^{l}=1$ as follows as a corollary of proof of Theorem 4.1 (see Remark 5.8). In particular, for minimal, Gorenstein $X$ of Albanese dimension $(n-1)$, the inequality $K_{X}^{n} \geq 2(n-1) ! \chi\left(\omega_{X}\right)$ also holds.

Proof. Corollary $C$ (i) If $b=0$ just add up the Severi inequalities for $K_{X}$ and for $K_{F}$

$$
K_{f}^{n}=K_{X}^{n}+2 n K_{F}^{n-1} \geq 2 n ! \chi\left(\omega_{X}\right)+4 n ! \chi\left(\omega_{F}\right)=2 n !\left(\chi\left(\omega_{X}\right)-\chi\left(\omega_{F}\right) \chi\left(\omega_{B}\right)\right)=2 n ! \chi_{f}
$$

(ii) If $b \geq 1$ then $\omega_{f}$ is subcanonical and so $\delta\left(\omega_{f}\right)=2$. Then apply Proposition 2.7 and Theorem 4.1.

Main Theorem may be an useful tool for classification of irregular varieties. For example, we present here a stronger version of Severi inequality for surfaces with numerically decomposable canonical bundle, which is useful for the classification of irregular surfaces of small invariants (see for example [8] for recent progress). 
Proposition 4.6. Let $S$ be a smooth surface of maximal Albanese dimension such that $K_{S} \equiv L_{1}+L_{2}$ with $L_{i}$ nef line bundles. Then

$$
K_{S}^{2} \geq 4 \chi\left(\omega_{S}\right)+4 h_{a}^{1}\left(L_{1}\right)
$$

Proof. Let $\rho \in \operatorname{Pic}^{\tau}(S)$ such that $L_{1}+\left(L_{2}+\rho\right)=K_{S}$ and redefine $L_{2}=L_{2}+\rho$. Observe that $h_{a}^{i}\left(L_{1}\right)=h_{a}^{2-i}\left(L_{2}\right)$ and both are (numerically) sub-canonical. Applying Theorem (4.1) to both sheaves and adding up, we obtain

$$
L_{1}^{2}+L_{2}^{2} \geq 4\left(h_{a}^{0}\left(L_{1}\right)+h_{a}^{0}\left(L_{2}\right)\right)=4 \chi\left(S, L_{1}\right)+4 h_{a}^{1}\left(L_{1}\right)=2 L_{1}\left(-L_{2}\right)+4 \chi\left(\omega_{S}\right)+4 h_{a}^{1}\left(L_{1}\right)
$$

and hence

$$
K_{S}^{2}=L_{1}^{2}+2 L_{1} L_{2}+L_{2}^{2} \geq 4 \chi\left(\omega_{S}\right)+4 h_{a}^{1}\left(L_{1}\right)
$$

\section{Proof of Main Theorem}

As pointed out in the introduction, the proof relies on three basic tools: Xiao's method, the behavior of linear systems on suitable étale coverings (studied in Section 3) and finally Pardini's covering trick.

\subsection{Xiao's method}

We will use a simplified version of Xiao's method for fibrations in the case where the base curve is $\mathbb{P}^{1}$, and specially adapted to an ulterior process of étale covers. We remind briefly the method in this simplified version and refer to [2], [13], [23] and [29] for details. The construction holds in general for $\mathbb{Q}$-Cartier Weil divisors but for simplicity we state it for Cartier divisors, which is the case we will use.

Let $X$ be a normal projective variety of dimension $n$ and let $D$ be a nef Cartier divisor. Let $L=\mathcal{O}_{X}(D)$ be its associated line bundle. Assume we have a fibration $f: X \longrightarrow \mathbb{P}^{1}$ with $F$ a general fibre of $f$ and let $\mathcal{E}=f_{*} L$. It is a vector bundle since it is torsion free on a smooth curve. Consider its decomposition

$$
\mathcal{E}=f_{*} L=\bigoplus_{i=1}^{l} \mathcal{O}_{\mathbb{P}^{1}}\left(a_{i}\right)
$$

with $a_{1} \geq a_{2} \geq \ldots \geq a_{m} \geq 0>a_{m+1} \geq \ldots \geq a_{l}, l \geq m \geq 0$, and $l=h^{0}\left(F, L_{\mid F}\right)$. Observe that we have $h^{0}(X, L)=a_{1}+\ldots+a_{m}+m$ and so

$$
a_{1}+\ldots+a_{m} \geq h^{0}(X, L)-h^{0}\left(F, L_{\mid F}\right) .
$$

For $i=1, \ldots, m$, define $\mathcal{E}_{i}=\mathcal{O}_{\mathbb{P}^{1}}\left(a_{1}\right) \oplus \ldots \oplus \mathcal{O}_{\mathbb{P}^{1}}\left(a_{i}\right)$. When the $a_{i}$ 's are different, these are the pieces of the Harder-Narashiman filtration of $\mathcal{E}_{m}$, of associated slopes $\mu_{i}=a_{i}$.

For each $i=1, \ldots, m$ such that $a_{i}>a_{i+1}$ the composite of the natural sheaf homomorphisms

$$
f^{*} \mathcal{E}_{i}\left(-a_{i}\right) \rightarrow f^{*}\left(f_{*} L\right)\left(-a_{i}\right) \rightarrow L\left(-a_{i} F\right)
$$

surjects onto a sheaf of ideals of type $\mathcal{I}_{Z_{i}} \otimes L\left(-a_{i} F\right)$. Following [23] Lemma 1.1 and Remark therein, up to a suitable desingularization $\epsilon: \widehat{X} \longrightarrow X$, if we set $\widehat{L}=\epsilon^{*} L$ and $\widehat{F}=\epsilon^{*} F$, we have a decomposition

$$
\widehat{L}=N_{i}+\widehat{Z}_{i}+a_{i} \widehat{F}
$$

where: 
- $N_{i}$ is a nef Cartier divisor on $\widehat{X}$ inducing a base point free linear system.

- $\widehat{Z}_{i}$ is an effective and fixed Cartier divisor (the base divisor of the induced linear system on $\widehat{X}$ ).

If $a_{i}=a_{i+1}$ we define $N_{i}=N_{i+1}, \widehat{Z}_{i}=\widehat{Z}_{i+1}$. Observe that $N_{1}=\mathcal{O}_{\widehat{X}}$ if and only if $a_{1}>a_{2}$. We redefine $a_{m+1}=0$ and extend coherently the definition to $N_{m+1}$ and $\widehat{Z}_{m+1}$.

Moreover, we have that

$$
\begin{gathered}
N_{1} \leq N_{2} \leq \ldots \leq N_{m} \leq N_{m+1} \leq L \\
\widehat{Z}_{1} \geq \widehat{Z}_{2} \geq \ldots \geq \widehat{Z}_{m} \geq \widehat{Z}_{m+1} \geq 0 \\
a_{1} \geq a_{2} \geq \ldots \geq a_{m} \geq a_{m+1}=0
\end{gathered}
$$

In fact, by construction we have that

$$
N_{i}+\left(\widehat{Z}_{i}-\widehat{Z}_{m+1}\right)=N_{m+1}\left(-a_{i} \widehat{F}\right)
$$

is the decomposition of $N_{m+1}\left(-a_{i} \widehat{F}\right)$ in its moving and fixed part, respectively.

Under these assumptions we can apply Xiao's Lemma (see [13]) and [23] Lemma 1.2. We define the linear systems $P_{i}:=\left.N_{i}\right|_{\widehat{F}}$ which are free from base points and induce maps $\phi_{i}: \widehat{F} \longrightarrow \mathbb{P}^{r_{i}-1}$. Observe that for $i=1, \ldots, m$ we have $r_{i} \geq i$.

Define now

$$
I_{s}=\left\{k=1, \ldots, m \mid \operatorname{dim} \phi_{k}(\widehat{F})=s\right\}
$$

and we obtain a partition of the set $\{1, \ldots, m\}$.

Let $r$ be the maximum index such that $I_{r-1} \neq \emptyset$ and define decreasingly, for $s=1, \ldots, r-1$

$$
b_{s}=\left\{\begin{array}{cc}
\min I_{s} & \text { if } I_{s} \neq \emptyset \\
b_{s+1} & \text { otherwise }
\end{array}\right.
$$

Then we have that, for any $A_{1}, \ldots, A_{n-r}$ nef Cartier divisors the following inequality holds:

$$
A_{1} \ldots A_{n-r}\left[N_{m+1}^{r}-\left(\sum_{s=r-1}^{1}\left(\prod_{k>s} P_{b_{k}}\right) \sum_{i \in I_{s}}\left(\sum_{l=0}^{s} P_{i}^{s-l} P_{i+1}^{l}\right)\left(a_{i}-a_{i+1}\right)\right)\right] \geq 0 .
$$

In particular, taking $A_{1}=\ldots=A_{n-r}=\widehat{L}$ we obtain

$$
L^{n}=(\widehat{L})^{n} \geq \widehat{L}^{n-r} N_{m+1}^{r} \geq \widehat{L}^{n-r}\left[\sum_{s=r-1}^{1}\left(\prod_{k>s} P_{b_{k}}\right) \sum_{i \in I_{s}}\left(\sum_{l=0}^{s} P_{i}^{s-l} P_{i+1}^{l}\right)\left(a_{i}-a_{i+1}\right)\right]
$$

Since $P_{i+1} \geq P_{i}$ and they are nef, we have that

$$
\sum_{l=0}^{s}\left(P_{i}^{s-l} P_{i+1}^{l}\right) \geq(s+1) P_{i}^{s}
$$

and so

$$
L^{n} \geq \widehat{L}^{n-r}\left[\sum_{s=r-1}^{1}(s+1)\left(\prod_{k>s} P_{b_{k}}\right) \sum_{i \in I_{s}} P_{i}^{s}\left(a_{i}-a_{i+1}\right)\right]
$$

For later reference we need to consider the following special case. Assume that all the induced maps $\phi_{i}$ have image of dimension $n-1$, i.e., they are generically finite. Then 


$$
\left.L^{n} \geq n\left[P_{m}^{n-1}\left(a_{m}-a_{m+1}\right)+P_{m-1}^{n-1}\left(a_{m-1}-a_{m}\right)+\ldots+P_{1}^{n-1}\left(a_{1}-a_{2}\right)\right)\right]
$$

Remark 5.4. The same construction can be applied to any selection of indexes $I \subseteq\{1, \ldots, m\}$.

We are going to see now how the method behaves under a suitable étale Galois covering of $X$. It turns out that a continuous Xiao's method holds.

Proposition 5.5. Let $X$ be a normal projective variety and $L$ a line budle. Let $a: X \longrightarrow A$ be a nontrivial map to an abelian variety and $f: X \longrightarrow \mathbb{P}^{1}$ a fibration. Then, for a very general element $\beta \in \widehat{A}, L^{\prime}:=L \otimes \beta$ verifies that the vector bundles $\mathcal{E}_{\alpha}=f_{*}\left(L^{\prime} \otimes \alpha\right)$ are all equal for $\alpha \in \widehat{A}_{\text {tors }}$.

Proof. The continuous family of vector bundles $\left\{f_{*}(L \otimes \beta)\right\}$ on $\mathbb{P}^{1}$ for $\beta \in \widehat{A}$ must be constant on a nonempty open set $U$. Let $D=\widehat{A} \backslash U$. Then

$$
\bigcup_{\alpha \in \widehat{A}_{\text {tors }}}(\alpha+D) \neq \widehat{A}
$$

being a countable union of proper closed sets. An element $\beta$ in its complementary set verifies the statement.

Consider a fibration $f: X \longrightarrow \mathbb{P}^{1}$ and a nontrivial map $a: X \longrightarrow A$ such that $a^{*}$ is injective. Let $L$ be a nef line bundle such that $h_{a}^{0}(L) \neq 0$ and which verifies the conclusion of Proposition 5.5, i.e., for all $\alpha \in \widehat{A}_{\text {tors }}$, the sheaves $f_{*}(L \otimes \alpha)$ are all equal. Keeping all the previous notations, for $i=1, \ldots, m$ consider the linear systems $L\left(-a_{i} F\right)$. Apply now Theorem 3.3 to all of them and get

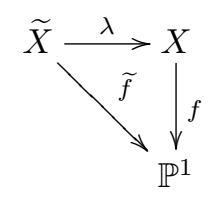

where $\lambda$ is a composition of a blow-up and an étale Galois map induced by $\widehat{A}_{d}$ for certain $d$, verifying the following conditions

- For $i=1, \ldots, m$ and for any $\alpha \in \widehat{A}$ we have $\lambda^{*}\left(L\left(-a_{i} F\right)\right) \otimes \alpha=W_{i}+N_{\alpha, i}$.

- $W_{i}$ does not depend on $\alpha$ and is the fixed component of the linear system.

- $N_{\alpha, i}$ induce a base point free linear system and for all $\alpha$ we have $N_{\alpha, i}=N_{0, i} \otimes \alpha$.

Furthermore, since the kernel of the map $\operatorname{Pic}^{0}(X) \longrightarrow \operatorname{Pic}^{0}(F)$ is finite, we can choose $d$ in such a way that $\widetilde{F} \equiv \lambda^{*} F$ is a connected étale cover of $F$ and hence $\widetilde{f}$ is a fibration.

Let $\widetilde{L}=\lambda^{*} L$, and $r=\operatorname{deg} \mu$. By the choice of $L$ and projection formula we have

$$
\widetilde{\mathcal{E}}=\widetilde{f}_{*} \widetilde{L}=\mathcal{E}^{\oplus r}=\mathcal{O}_{\mathbb{P}^{1}}\left(a_{1}\right)^{\oplus r} \oplus \ldots \oplus \mathcal{O}_{\mathbb{P}^{1}}\left(a_{l}\right)^{\oplus r}
$$

Applying Xiao's method to $\widetilde{f}$ we have that the vector bundles $\widetilde{\mathcal{E}}_{j}$ induce base point free linear systems $\widetilde{N}_{j}$ on $\widetilde{X}$ and $\widetilde{P}_{j}$ on $\widetilde{F}$. Among these we have for $i=1, \ldots, m$

$$
\widetilde{\mathcal{E}}_{r i}=\mathcal{O}_{\mathbb{P}^{1}}\left(a_{1}\right)^{\oplus r} \oplus \ldots \oplus \mathcal{O}_{\mathbb{P}^{1}}\left(a_{i}\right)^{\oplus r}
$$

which induce precisely the linear systems $\widetilde{N}_{r i}=N_{0, i}$ defined above. Then $\widetilde{P}_{r i}=N_{0, i \mid \widetilde{F}}$ which by construction has a space of sections of dimension at least $\operatorname{rank} \widetilde{E}_{r i}=r i$. Following the previous conventions recall that if $r(i-1)<j \leq$ ri $i$ then $\widetilde{P}_{j}=\widetilde{P}_{r i}$.

Summing up we obtain 
Proposition 5.6. $\quad$ (i) $h_{\widetilde{a}}^{0}\left(\widetilde{P}_{r i}\right) \geq r i$.

(ii) If $X$ is of maximal a-dimension, we can choose $\lambda$ in such a way that for all $j=1, \ldots, r m$ the linear systems $\left|\widetilde{P}_{j}\right|$ are generically finite.

Proof. (i) We can do the same construction for $\widetilde{\mathcal{E}}_{\alpha}=\widetilde{f}_{*}(\widetilde{L} \otimes \alpha)$. Similarly we obtain linear systems $\widetilde{N}_{r i}^{\alpha}=N_{\alpha, i}$ such that when restricted to $\widetilde{F}$ they induce $\widetilde{P}_{r i}^{\alpha}$ of dimension at least ri. By construction for any $\alpha$ we have that $N_{\alpha, i}=N_{0, i} \otimes \alpha$ and hence the same happens when restricting to $\widetilde{F}$. Hence for all $\alpha$ we have that $h^{0}\left(\widetilde{F}, \widetilde{P}_{r i} \otimes \alpha\right)=h^{0}\left(\widetilde{F}, \widetilde{P}_{r i}^{\alpha}\right) \geq r i$ and so $h_{\widetilde{a}}^{0}\left(\widetilde{P}_{r i}\right) \geq r i$.

(ii) We have that $\widetilde{P}_{j}=\widetilde{P}_{r i}$ for some $i$. By Theorem 3.5 we can modify $\lambda$ by a multiplication map with $d>>0$ such that the maps induced by $N_{\alpha, i}$ are all generically finite.

\subsection{The proof}

We will use freely the notations of subsection 5.1. Observe that if $h_{a}^{0}(L)=0$ then the result is trivially true. From now on we will consider that $h_{a}^{0}(L) \neq 0$. We can also assume that the map $a^{*}: \widehat{A} \longrightarrow \operatorname{Pic}^{0} X$ is injective, and hence apply freely the results of Section 3 and Subsection 5.1. Indeed, consider the abelian variety $B=\operatorname{Im}\left(a^{*}\right)$ and let $C=\widehat{B}$. We have a factorization of $a$

$$
X \stackrel{\operatorname{alb}_{X}}{\longrightarrow} \operatorname{Alb}_{X} \stackrel{\pi}{\longrightarrow} C \longrightarrow A
$$

with $\left(\pi \circ \operatorname{alb}_{X}\right)^{*}: \widehat{C}=B \longrightarrow \operatorname{Pic}^{0} X$ injective and $h_{a}^{0}(L)=h_{\pi \circ a^{\prime} b_{X}}^{0}(L)$. Since the map $C \longrightarrow A$ is étale onto the image of $a$, clearly the $a$-dimension of $X$ coincides with the $\left(\pi \circ \operatorname{alb}_{X}\right)$-dimension of $X$.

Observe that the injectivity property is stable by restriction to any $M$, a big and nef smooth divisor on $X$, since $\mathrm{Pic}^{0} X \cong \operatorname{Pic}^{0} M$ if $\operatorname{dim} M \geq 2$ and $\operatorname{Pic}^{0} X \subseteq \operatorname{Pic}^{0} M$ if $\operatorname{dim} M=1$.

(i) Assume that $X$ is of maximal $a$-dimension. We proceed by induction on $n=\operatorname{dim} X$.

Case $n=1$. Let $X$ be a smooth curve of genus $g \geq 1$ and $L$ a nef line bundle. If $\operatorname{deg} L \leq \operatorname{deg} K_{X}=2 g-2$ and it is non-special, then $\operatorname{deg} L \geq 2 h^{0}(X, L)$ by Riemann-Roch theorem. If $\operatorname{deg} L=r(2 g-2)$ with $r \in \mathbb{Q}, r>1$ then again by Riemann-Roch theorem we obtain $\operatorname{deg} L=\frac{2 r}{2 r-1} h^{0}(X, L)$. It remains the case of an special divisor. Take the étale cover of $C$ induced by $\widehat{A}_{d}$, say $\mu: \widetilde{C} \longrightarrow C$. Consider $\widetilde{L}=\mu^{*} L$ which is an special line bundle on $\widetilde{C}$. Hence, we can apply Clifford's theorem and obtain

$$
d^{2 q} \operatorname{deg} L=\operatorname{deg} \widetilde{L} \geq 2 h^{0}(\widetilde{C}, \widetilde{L})-2 \geq 2 d^{2 q} h_{a}^{0}(L)-2
$$

where $q=\operatorname{dim} A$. Since this holds for all $d$ we obtain $\operatorname{deg} L \geq 2 h_{a}^{0}(L)$.

Case $n \geq 2$. We assume now that for any irregular variety $X^{\prime}$ of dimension at most $n-1$, with a nontrivial map $a^{\prime}: Y \longrightarrow A^{\prime}$ to an abelian variety, and for any nef line bundle $L^{\prime}$, inequality (i) holds. The key argument here is to use this induction hypothesis in Xiao's method on a suitable étale cover of $X$ to prove an inequality (Step 1) from which we can apply the main idea of Pardini's proof in [24] (Step 2).

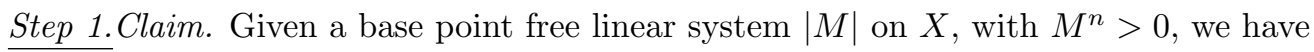

$$
L^{n}+n L^{n-1} M \geq \delta(L) n ! h_{a}^{0}(L) .
$$

Proof of Claim. The general member of $|M|$ is smooth and irreducible. Take two general smooth members $F, F^{\prime} \in|M|$. Consider a blow up $\epsilon: Y \longrightarrow X$ in order to get a fibration $f: Y \longrightarrow \mathbb{P}^{1}$ induced by $F$ and $F^{\prime}$. Since the formula we want to prove is invariant in the algebraic class of $L$, we change $L$ by $L \otimes \beta$ 
in such a way that Proposition 5.5 applies for $\epsilon^{*} L$. Moreover we can get that $h^{0}(X, L)=h_{a}^{0}(L)$ and that $h^{0}\left(F, L_{\mid F}\right)=h_{a}^{0}\left(L_{\mid F}\right)$.

Then we can apply the construction of Subsection 5.1 to $f: Y \longrightarrow \mathbb{P}^{1}$ and get

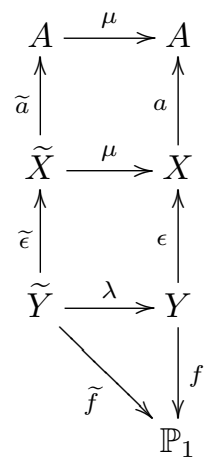

where $\mu$ is of degree $r=d^{2 q}(q=\operatorname{dim} A)$. Let $\widetilde{L}=\lambda^{*}\left(\epsilon^{*} L\right)$ and $\widetilde{F}=\lambda^{*} F$, which is an irreducible étale cover of $F$ of degree $r$. We can apply Proposition 5.6 (ii) and so all the induced maps are generically finite and hence by (5.3) we obtain

$$
\widetilde{L}^{n} \geq n\left[\widetilde{P}_{r}^{n-1}\left(a_{1}-a_{2}\right)+\ldots+\widetilde{P}_{r m}^{n-1} a_{m}\right] .
$$

Observe that $\widetilde{F}$ is $(n-1)$-dimensional, of maximal $\widetilde{a}_{\mid \widetilde{F}^{-}}$-dimension and the map $\widetilde{a}_{\mid \widetilde{F}}$ is non trivial. So we apply the induction hypothesis for the nef line bundles $\widetilde{P}_{i}$ on $\widetilde{F}$, and for all $i=1, \ldots, m$ we get

$$
\widetilde{P}_{r i}^{n-1} \geq \delta\left(\widetilde{P}_{r i}\right)(n-1) ! h_{\widetilde{a}}^{0}\left(\widetilde{P}_{r i}\right) \geq \delta(L)(n-1) ! h_{\widetilde{a}}^{0}\left(\widetilde{P}_{r i}\right)
$$

the last inequality holding by Remark 2.10. By Proposition 5.6 (i) we have that

$$
h_{\widetilde{a}}^{0}\left(\widetilde{P}_{r i}\right) \geq r i
$$

Thus

$$
r L^{n}=\widetilde{L}^{n} \geq r \delta(L) n !\left(a_{1}+\ldots+a_{m}\right) \geq r \delta(L) n !\left(h_{a}^{0}(L)-h_{a}^{0}\left(\epsilon^{*} L_{\mid F}\right)\right)
$$

Using again induction for $\epsilon^{*} L_{\mid F}$ we have

$$
\left(\epsilon^{*} L_{\mid F}\right)^{n-1} \geq \delta\left(\epsilon^{*} L_{\mid F}\right)(n-1) ! h_{a}^{0}\left(\epsilon^{*} L_{\mid F}\right) \geq \delta(L)(n-1) ! h_{a}^{0}\left(\epsilon^{*} L_{\mid F}\right)
$$

and summing up, since $\epsilon_{*} F=M$ we finally obtain

$$
L^{n}+n L^{n-1} M \geq \delta(L) n ! h_{a}^{0}(L) .
$$

Step 2. Let us apply now Pardini's covering trick to prove the statement. Consider again $d \in \mathbb{N}$ and the étale Galois map induced by multiplication

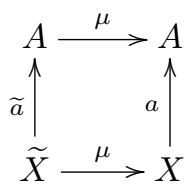

Let $H$ be a fixed very ample line bundle on $A$ and let $M=a^{*} H, \widetilde{M}=\widetilde{a}^{*}(H)$. By [14] Ch2. Prop. 3.5 we have 


$$
\mu^{*} H \equiv d^{2} H
$$

and so

$$
\widetilde{M} \equiv \frac{1}{d^{2}} \mu^{*} M
$$

Define again

$$
\widetilde{L}:=\mu^{*} L
$$

We have

- $h_{\widetilde{a}}^{0}(\widetilde{L})=d^{2 q} h_{a}^{0}(L)$ by Proposition 2.8, and $\delta(\widetilde{L})=\delta(L)$ by Remark 2.10 .

- For all $i=0, \ldots, n \widetilde{L}^{n-i} \widetilde{M}^{i}=d^{2 q-2 i} L^{n-i} M^{i}$ by (5.7).

We can apply now the Claim of Step 1 to $(\widetilde{M}, \widetilde{X}, \widetilde{L})$ :

$$
(\widetilde{L})^{n}+n(\widetilde{L})^{n-1} \widetilde{M} \geq \delta(\widetilde{L}) n ! h_{a}^{0}(\widetilde{L})
$$

And hence:

$$
d^{2 q} L^{n}+n d^{2 q-2} L^{n-1} M \geq d^{2 q} \delta(L) n ! h_{a}^{0}(L)
$$

which holds for all $d$. Thus we can conclude

$$
L^{n} \geq \delta(L) n ! h_{a}^{0}(L) .
$$

(ii) Assume now that $1 \leq k=\operatorname{dim} a(X)<n$ and that $M_{\mid G}$ is big, $G$ being a general fibre of the algebraic fibre space induced by $a$ and $M$ the continuous moving part of the linear system $|L|$.

Following Theorem 3.6 (ii), up to a composition of a blow-up and an étale cover, we have $\lambda^{*} L=W+N$ with $|N|$ base point free and generically finite, $h_{a}^{0}(N)=(\operatorname{deg} \mu) h_{a}^{0}(L)$ and $\delta(N) \geq \delta(L)$ (see Remark 2.10). Since $L$ and $N$ are nef we have $(\operatorname{deg} \mu) L^{n}=\left(\lambda^{*} L\right)^{n} \geq N^{n}$. Hence its enough to prove the statement for $N$.

Take general elements $N_{1}, \ldots N_{n-k} \in|N|$ and let $T=N_{1} \cap \ldots \cap N_{n-k}$. We have that $T$ is smooth, $k$ dimensional and dominates $a(X)$ since $N$ is transversal to a general $G$ (it induces a generically finite map). Hence $T$ is of maximal $a$-dimension. Let $N_{T}=N_{\mid T}$. We have $h_{a}^{0}\left(N_{T}\right) \geq h_{a}^{0}(N)$ (by Proposition 2.8 (iii)) and $\delta\left(N_{T}\right) \geq \delta(N)$ (by Remark 2.10). Then we apply (i) to the pair $\left(T, N_{T}\right)$ with respect to $a$ and obtain

$$
N^{n}=\left(N_{T}\right)^{k} \geq \delta\left(N_{T}\right) k ! h_{a}^{0}\left(N_{T}\right) \geq \delta(N) k ! h_{a}^{0}(N) .
$$

(iii) As in (ii) up to an étale cover and blow-up we obtain $\left|\lambda^{*} L\right|=W+|N|$. But in this case $N=\phi^{*} R$ where $\phi: \widetilde{X} \longrightarrow Z$ is the algebraic fibre space induced by $|N|, \operatorname{dim} Z \geq k$ and the linear system $|R|$ on $Z$ is generically finite. Up to blow-ups on $\widetilde{X}$ and $Z$ we can assume that $Z$ is smooth. $L$ is big so also $\lambda^{*} L$ is. Contrary to (ii), in this case there is no clear relation between $\delta(R)$ and $\delta(N) \geq \delta\left(\lambda^{*} L\right) \geq \delta(L)$, so we only use $\delta(R) \geq 1$.

Let $r=\operatorname{dim} Z$ and let $\bar{G}$ be the fibre of $\phi$. By nefness of $\lambda^{*} L$ and $N$ and bigness of $\left(\lambda^{*} L\right)_{\mid G}$ we have

$$
L^{n}=\left(\lambda^{*} L\right)^{n} \geq\left(\lambda^{*} L\right)^{n-r} \phi^{*}(R)^{r}=\left(\left(\lambda^{*} L\right)_{\bar{G}}\right)^{n-r} R^{r} \geq R^{r} .
$$

If $\operatorname{dim} Z=k$ we just apply (i) to the pair $(Z, R)$. If $\operatorname{dim} Z>k$, since $|R|$ is base point free on $Z$ and induces a generically finite map, we can apply to it the same argument as in (ii). In any case we obtain

$$
R^{r} \geq \delta(R) k ! h_{\tilde{a}}^{0}(R) \geq k ! h_{a^{\prime}}^{0}(R)=k ! h_{a}^{0}(L) .
$$


Remark 5.8. As a corollary of proof, observe that in the proof of (iii) we can obtain the same inequality as in (ii) provided that $\left(\left(\lambda^{*} L\right)_{\bar{G}}\right)^{n-r} \geq 2$. In other words, if (ii) does not hold, then $\widetilde{X}$ is fibred by a family of varieties $\bar{G}$ with $\left(\left(\lambda^{*} L\right)_{\bar{G}}\right)^{n-r}=1$.

When $X$ is Gorenstein and minimal, $L=K_{X}$ and $a=a l b_{X}$, observe that this implies that the $\widetilde{a}$-fibres of $\widetilde{X}$ are fibred by $\bar{G}$ such that $K_{\bar{G}}^{n-r}=1$. Since the general fibre of the algebraic fibre space induced by $\widetilde{a}$ is isomorphic to the general fibre of the algebraic fibre space induced by $a l b_{X}$ on $X$, then we can conclude that if $K_{X}^{n}<2 k ! \chi\left(\omega_{X}\right)$, then $a l b_{X}$ factors through a fibration with general fibre $G$ such that $K_{G}^{\operatorname{dim} G}=1$. Observe that this cannot happen when $\operatorname{dim} \operatorname{alb}(X)=n-1$.

\section{References}

[1] M.A. Barja, M. Lahoz, J.C. Naranjo, G. Pareschi, On the bicanonical map of irregular varieties, J. Algebraic Geom. 213 (2012), 445-471.

[2] M.A. Barja, L. Stoppino, Stability conditions and positivity of invariants of fibrations, arXiv:1212.4769.

[3] M. Beltrametti, A. Sommese, The adjuntion theory of complex projective varieties, De Grutyer Expositions in Mathematics 16 (1991).

[4] F. Catanese, Moduli of surfaces of general type, Algebraic Geometry: open problems. Proceedings Ravello 1982, Springer-Verlag L.N.M. 997 (1983), 90-112.

[5] F. Catanese, C. Ciliberto, M. Mendes-Lopes, On the classification of irregular surfaces of general type with nonbirational bicanonical map, Trans. Amer. Math. Soc. 3501 (1998), 275-308.

[6] M. Chen, A sharp lower bound for the canonical volume of 3-folds of general type, Math. Ann. 337 (2007), 165-181.

[7] J. A. Chen, M. Chen, The canonical volume of 3-folds of general type with $\chi \leq 0$, J. Lond. Math. Soc. 2 783 (2008), 693 - 706.

[8] C. Ciliberto, M. Mendes-Lopes, R. Pardini, The classification of minimal irregular surfaces of general type with $K_{S}^{2}=2 p_{g}$, arXiv: 1307.6228 .

[9] M. Cornalba, J. Harris, Divisor classes associated to families of stable varieties, with applications to the moduli space of curves, Ann. Sc. Ec. Norm. Sup. 214 (1988), 455-475.

[10] O. Debarre, On coverings of simple abelian varieties, Bull. Soc. Math. France 1342 (2006), 253-260.

[11] T. Fujita, On Kahler fiber spaces over curves, J. Math. Soc. Japan 30 (1978), 779-794.

[12] C. Hacon, R. Pardini, Birational classification of curves of genus 2, Math. Res. Let. 121 (2005), 129-140.

[13] K. Konno, A lower bound of the slope of trigonal fibrations, Internat. J. Math. 7 (1) (1996), 19-27.

[14] H. Lange, C. Birkenhake, Complex Abelian Varieties, G. m. W. Springer-Verlag 302 (1992).

[15] M. Manetti, Surfaces of Albanese general type and the Severi conjecture, Math. Nach. 261-262 (2003), $105-122$.

[16] M. Mendes-Lopes, R. Pardini, Severi type inequalities for irregular surfaces with ample canonical class, Comment. Math. Helv. 862 (2011), 401-414.

[17] M. Mendes-Lopes, R. Pardini, The geography of irregular surfaces, Current developements in algebraic geometry, MSRI Publications 59 (2011), 349-378.

[18] M. Mendes-Lopes, R. Pardini, G.P. Pirola, Continuous families of divisors, paracanonical systems and a new inequality for varieties of maximal Albanese dimension, Geom. Topol. 17 (2) (2013), 1205-1223. 
[19] M. Mendes-Lopes, R. Pardini, G.P. Pirola, Brill-Noether loci for divisors on irregular varieties, mathAG:1112.6357.

[20] Y. Miyaoka, The Chern classes and Kodaira dimension of a minimal variety, Algebraic Geometry, Sendai 1985; Adv. Studies in Pure Math. 10 (1987), 449-476.

[21] D. Mumford, On the equations defining abelian varieties, I, Invent. Math. 4 (1966), 287-354.

[22] D. Mumford, Varieties defined by quadratic equations, Questions on Algebraic Varieties, "C.I.M.E. Summer Schools" series 51 (1969), 29-100.

[23] K. Ohno, Some inequalities for minimal fibrations of surfaces of general type over curves, J. Math. Soc. Japan 444 (1992), 643-666.

[24] R. Pardini, The Severi inequality $K_{S}^{2} \geq 4 \chi\left(\omega_{S}\right)$ for surfaces of maximal Albanese dimension, Invent. Math. 1593 (2005), 669-672.

[25] G. Pareschi, Basic results on irregular varieties via Fourier-Mukai methods, Current Developments in Algebraic Geometry, MSRI Publications 59 (2011), 379-403.

[26] G. Pareschi, M. Popa, Regularity on abelian varieites I, Journal Amer. Math. Soc. 162 (2003), 285-302.

[27] G. Pareschi, M. Popa, GV sheaves, Fourier-Mukai transforms and Generic Vanishing, Amer. J. Math. 1331 (2011), 235-271.

[28] F. Severi, La serie canonica e la teoria delle serie principali di gruppi di punti supra una superficie algebrica, Comm. Math. Helv. 4 (1932), 268-326.

[29] G. Xiao, Fibred algebraic surfaces with low slope, Math. Ann. 276 (1987), 449-466.

[30] L. Zhang, An orbifold approach to Severi inequality, mathAG:1202.2656.

[31] T. Zhang, Severi inequality for varieites of maximal Albanese dimension, mathAG:1303.4043.

Miguel Ángel Barja

Departament de Matemàtica Aplicada I, ETSEIB-Facultat de Matemàtiques i Estadística

Universitat Politècnica de Catalunya-BarcelonaTECH

Avda. Diagonal, 647

08028 Barcelona (Spain).

e-mail: miguel.angel.barja@upc.edu 